\title{
Estimation of the Reliability Measures of a Three - Component System with Human Errors and Common Cause Failures
}

\author{
B. R. Sreedhar \\ Assistant Professor \\ Dept. Of Mathematics \\ Chaitanya Bharathi \\ Institute of Technology \\ Hyderabad, India
}

\author{
K.Pushpanjali \\ Professor \\ Department Of Statistics \\ Sri Krishnadevaraya \\ University \\ Anantapur, A.P., India
}

\author{
G. Y. Sagar \\ Associate Professor \\ Dept. Of Mathematics \\ T R R Engineering \\ College, \\ Hyderabad, India.
}

\author{
Y. R. Reddy \\ Teaching Assistant \\ Department Of Or\&Sqc \\ Rayalaseema University, \\ Kurnool, India
}

\begin{abstract}
The present paper discusses the problem of estimating the reliability measures of a three-component identical system when the system is affected by Common Cause Shock (CCS) failures as well as human errors. The maximum likelihood estimators of the reliability measures like reliability function and mean time between failures of the present model are obtained. The performances of the proposed estimates have been developed in terms of mean square error, using simulated data.
\end{abstract}

\section{General Terms}

Three-component identical system, Reliability measures, Reliability estimates, Maximum likelihood estimation.

\section{Keywords}

M L estimation, CCS failures, Human errors, Reliability function, MTBF, Monte-Carlo Simulation.

\section{INTRODUCTION}

In modern industries very high reliability systems are needed but it is universally accepted that computers cannot achieve the intended reliability in operating systems, application programs, control programs or commercial systems such as space shuttle, nuclear power plant control, etc., without employing redundancy. Further, there are other factors such as Common Cause Shock (CCS) failures and human errors etc. which could cause the whole system to fail. Therefore, the interest lies in assessing and estimating system performance measures like Reliability function, Mean time between failures (MTBF) etc. in the presence of the above said failures. However estimation methods and techniques were available for estimating the parameters and reliability of the system in the literature. Sagar et al [6] derived the reliability measures of a three component identical system with CCS failures and human errors. Billinton and Allan [2] discussed the role of Common cause failures in reliability modeling. Atwood [1] used the BFR model for Common cause failures in the area of nuclear power plants. Chari et al [3] derived the reliability measures of a two component identical system under the influence of CCS failures. Ritika wason [5] studied the traditional software reliability estimation. Reddy [4] derived reliability measures in the presence of lethal and nonlethal CCS failures of a two component non-identical system.
In this paper, we have tried to evaluate the estimation approach which could give formal estimation procedure of the reliability measures with specific reference to CCS failures as well as human errors. In this connection, we considered three-component identical system with CCS failures as well as human errors and the M L estimation approach is proposed to estimate the reliability measures of the present model.

Therefore, the purpose of this paper is not only to estimate the reliability measures but also to report on the results of our simulation study and to resolve differences among other existing studies of system reliability.

\section{NOTATIONS}

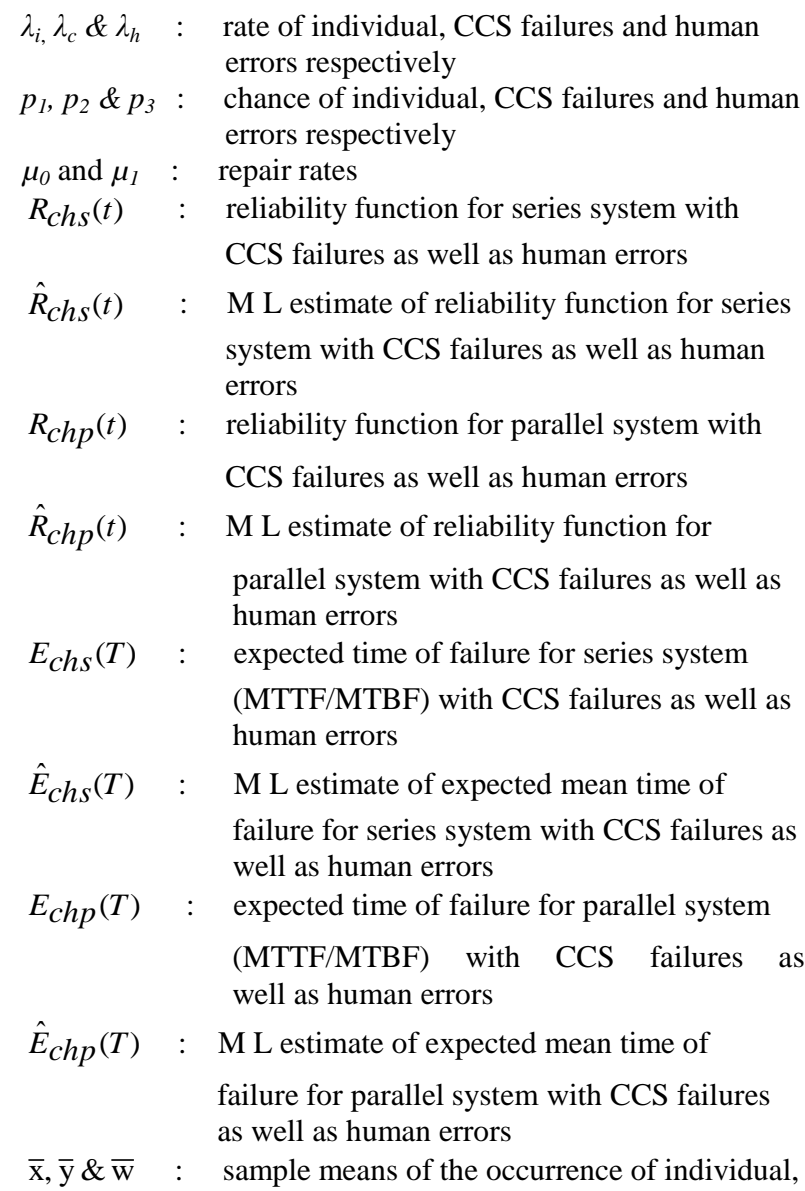


CCS failures and human errors respectively sample mean of service time of the components

$\hat{\overline{\mathrm{X}}}, \hat{\overline{\mathrm{y}}} \& \hat{\overline{\mathrm{W}}} \quad$ : $\quad$ sample estimates of individual failure rate, CCS failure rate and human errors respectively

$\hat{\bar{Z}} \quad$ : sample estimate of service time of the components

n : : sample size

$\mathrm{N} \quad: \quad$ number of simulated samples

M S E : mean square error

\section{ASSUMPTIONS}

(i) The system consists of three s-independent and identical components.

(ii) The system is affected by individual, CCS failures and human errors

(iii) The arrival stream of individual failures, CCS failures and human errors from a Poisson process with arrival rates $\lambda_{i}, \lambda_{c} \& \lambda_{h}$ respectively. The chance of such failures are $p_{1}, p_{2} \& p_{3}$ such that $p_{1}+p_{2}+p_{3}=1$.

(iv) The times between individual failures, CCS failures and human errors follow an exponential distribution.

(v) The individual failures, CCS failures and human errors occurs independent of each other.

(vi) The failed components are repaired singly and repair times follow an exponential distribution with rate of repair ' $\mu$ '.

\section{THE MODEL}

Keeping in view of the above assumptions, we formulate a Markov model to obtain the reliability function and MTBF of the system under the influence of individual, common cause shock failures as well as human errors. The Markovian graph is given in Fig. (4.1) and the quantities appear in Fig. (4.1) are to be read as

$\lambda_{0}=3 \lambda_{i} p_{1} ; \quad \lambda_{1}=2 \lambda_{i} p_{1} ; \quad \lambda_{2}=\lambda_{i} p_{1} ; \quad \lambda_{12}=\lambda_{c} p_{2} ; \lambda_{13}=\lambda_{h} p_{3} ;$ $\mu_{0}=\mu ; \mu_{l}=2 \mu$

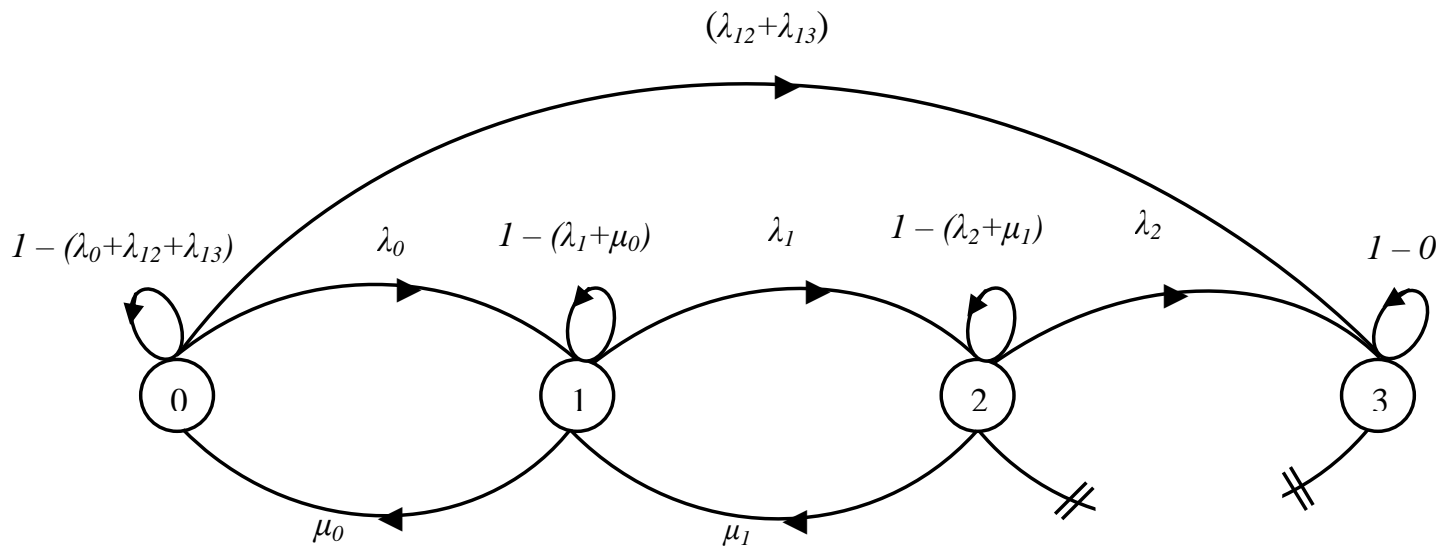

\section{Fig. 4.1 Markov Graph for Reliability Functions of Three-Component Identical System with Individual, CCS failures and Human errors}

The differential equations associated with the system states are

$p^{\prime}{ }_{0}=-\left(3 \lambda_{i} p_{1}+\lambda_{c} p_{2}+\lambda_{h} p_{3}\right) p_{0}(t)+p_{1}(t) \mu$

$p^{\prime}{ }^{\prime}=\left(3 \lambda_{i} p_{1}\right) p_{0}(t)-\left(2 \lambda_{i} p_{I}+\mu\right) p_{I}(t)+2 \mu p_{2}(t)$

$p_{2}^{\prime}=\left(2 \lambda_{i} p_{1}\right) p_{1}(t)-\left(\lambda_{i} p_{1}+2 \mu\right) p_{2}(t)$

$p_{3}^{\prime}=\left(\lambda_{c} p_{2}+\lambda_{h} p_{3}\right) p_{0}(t)+\left(\lambda_{i} p_{1}\right) p_{2}(t)$

Using the Laplace transformation, the set of equations in (1) can be solve with the help of the initial conditions given at $\mathrm{t}=0, p_{0}(t)=1$ and $p_{1}(t)=p_{2}(t)=p_{3}(t)=0$ and the solution is

$$
\begin{aligned}
p_{0}(t)= & {\left[\left(\gamma_{1}^{2}+\gamma_{1} K+L\right) /\left(\gamma_{1}-\gamma_{2}\right)\left(\gamma_{1}-\gamma_{3}\right)\right] \exp \left(\gamma_{1} t\right) } \\
& -\left[\left(\gamma_{2}^{2}+\gamma_{2} K+L\right) /\left(\gamma_{1}-\gamma_{2}\right)\left(\gamma_{2}-\gamma_{3}\right)\right] \exp \left(\gamma_{2} t\right) \\
& +\left[\left(\gamma_{3}^{2}+\gamma_{3} K+L\right) /\left(\gamma_{1}-\gamma_{3}\right)\left(\gamma_{2}-\gamma_{3}\right)\right] \exp \left(\gamma_{3} t\right)
\end{aligned}
$$

$$
\begin{aligned}
p_{I}(t)= & {\left[\left(3 \lambda_{i} p_{1}\right)\left(\gamma_{1}+\lambda_{i} p_{1}+2 \mu\right) /\left(\gamma_{1}-\gamma_{2}\right)\left(\gamma_{1}-\gamma_{3}\right)\right] \exp \left(\gamma_{1} t\right) } \\
& -\left[\left(3 \lambda_{i} p_{1}\right)\left(\gamma_{2}+\lambda_{i} p_{1}+2 \mu\right) /\left(\gamma_{1}-\gamma_{2}\right)\left(\gamma_{2}-\gamma_{3}\right)\right] \exp \left(\gamma_{2} t\right) \\
& +\left[\left(3 \lambda_{i} p_{1}\right)\left(\gamma_{3}+\lambda_{i} p_{1}+2 \mu\right) /\left(\gamma_{1}-\gamma_{3}\right)\left(\gamma_{2}-\gamma_{3}\right)\right] \exp \left(\gamma_{3} t\right)
\end{aligned}
$$

$p_{2}(t)=\left[6\left(\lambda_{i} p_{1}\right)^{2} /\left(\gamma_{1}-\gamma_{2}\right)\left(\gamma_{1}-\gamma_{3}\right)\right] \exp \left(\gamma_{1} t\right)$

$-\left[6\left(\lambda_{i} p_{1}\right)^{2} /\left(\gamma_{1}-\gamma_{2}\right)\left(\gamma_{2}-\gamma_{3}\right)\right] \exp \left(\gamma_{2} t\right)$

$+\left[6\left(\lambda_{i} p_{1}\right)^{2} /\left(\gamma_{1}-\gamma_{3}\right)\left(\gamma_{2}-\gamma_{3}\right)\right] \exp \left(\gamma_{3} t\right)$ $p_{3}(t)=1-\left[p_{0}(t)+p_{1}(t)+p_{2}(t)\right]$

Where

$$
\begin{aligned}
& K=\left(3 \lambda_{i} p_{1}+3 \mu\right) \\
& L=\left(2\left(\lambda_{i} p_{1}\right)^{2}+\lambda_{i} p_{1} \mu+2 \mu^{2}\right) \\
& \gamma_{1}=-\gamma \sin (\alpha)-S_{I} / 3 \\
& \gamma_{2}=\gamma \sin (\pi / 3+\alpha)-S_{1} / 3 \\
& \gamma_{3}=\gamma \sin (-\pi / 3+\alpha)-S_{I} / 3
\end{aligned}
$$

Here

$$
\begin{aligned}
& \gamma=(2 / 3)\left(S_{1}^{2}-3 S_{2}\right)^{1 / 2} \\
& \alpha=\sin ^{-1}\left(-4 q / \gamma^{3}\right) / 3 \\
& q=S_{3}-\left(S_{1} S_{2}\right) / 3+2 S_{1}{ }^{3} / 27
\end{aligned}
$$

and $S_{1}, S_{2} \& S_{3}$ are defined as follows

$$
\begin{aligned}
S_{1}= & \left(6 \lambda_{i} p_{1}+\lambda_{h} p_{3}+\lambda_{c} p_{2}+3 \mu\right) \\
S_{2}= & {\left[11\left(\lambda_{i} p_{1}\right)^{2}+3 \lambda_{h} p_{3} \lambda_{i} p_{1}+3 \lambda_{c} p_{2} \lambda_{i} p_{I}\right.} \\
& \left.+7 \lambda_{i} p_{1} \mu+3 \lambda_{h} p_{3} \mu+3 \lambda_{c} p_{2} \mu+2 \mu^{2}\right] \\
S_{3}= & {\left[6\left(\lambda_{i} p_{1}\right)^{3}+2\left(\lambda i p_{1}\right)^{2} \lambda_{h} p_{3}+2\left(\lambda_{i} p_{1}\right)^{2} \lambda_{c} p_{2}\right.} \\
& \left.+2 \lambda_{c} p_{2} \mu^{2}+\lambda_{i} p_{1} \lambda_{h} p_{3} \mu+\lambda_{i} p_{1} \lambda_{c} p_{2} \mu+2 \lambda_{h} p_{3} \mu^{2}\right]
\end{aligned}
$$

$\gamma_{1,} \gamma_{2}$ and $\gamma_{3}$ are always negative, $\forall \lambda_{i} \geq 0, \mathrm{p}_{1}, \mathrm{p}_{2} \& \mathrm{p}_{3} \in(0,1)$ 


\section{MAXIMUM LIKELIHOOD (M L) ESTIMATION OF THE RELIABILITY MEASURES}

This section discusses the Maximum likelihood estimation approach for estimating the reliability measures of three component identical series and parallel systems in the presence of individual, CCS failures as well as human errors.

Let $x_{1}, x_{2}, \ldots \ldots . x_{n}$ be a sample of ' $n$ ' number of times between individual failures which will obey exponential law.

Let $y_{1}, y_{2}, \ldots \ldots y_{n}$ be a sample of ' $n$ ' number of times between CCS failures which follow exponential as well.

Let $w_{1}, w_{2}, \ldots \ldots . w_{n}$ be a sample of ' $\mathrm{n}$ ' number of times between human errors which follow exponential as well.

Let $z_{1}, z_{2}, \ldots \ldots . . . z_{n}$ be a sample of ' $n$ ' number of times repair of the components with exponential population law.

$\hat{\bar{x}}, \hat{\bar{y}}, \hat{\bar{w}} \& \hat{\bar{z}}$ are the maximum likelihood estimates of individual failure rate $\left(\lambda_{i}\right)$, CCS failure rate $\left(\lambda_{c}\right)$, human errors rate $\left(\lambda_{h}\right)$ and repair rate ' $\mu$ ' of the system respectively.

Where,

$$
\begin{aligned}
& \hat{\bar{x}}=\frac{1}{\bar{x}} ; \hat{\bar{y}}=\frac{1}{\bar{y}} ; \hat{\bar{w}}=\frac{1}{\bar{w}} ; \hat{\bar{z}}=\frac{1}{\bar{z}} \text { and } \\
& \bar{x}=\frac{\sum x_{i}}{n} ; \bar{y}=\frac{\sum y_{i}}{n} ; \bar{w}=\frac{\sum w_{i}}{n} ; \bar{z}=\frac{\sum z_{i}}{n}
\end{aligned}
$$

are the sample estimates of the rate of individual failure times, rate of CCS failure times, rate of human error times and rate of repair times of the components respectively.

\subsection{Estimation of the Reliability function - Series system}

The reliability function for series system is obtained as $R_{c h s}(t)=p_{0}(t)$

$$
=R_{1} \exp \left(\gamma_{1} t\right)-R_{2} \exp \left(\gamma_{2} t\right)+R_{3} \exp \left(\gamma_{3} t\right)
$$

Where

$$
\begin{aligned}
& R_{1}=\left(\gamma_{1}^{2}+\gamma_{1} K+L\right) /\left(\gamma_{1}-\gamma_{2}\right)\left(\gamma_{1}-\gamma_{3}\right) \\
& R_{2}=\left(\gamma_{2}^{2}+\gamma_{2} K+L\right) /\left(\gamma_{1}-\gamma_{2}\right)\left(\gamma_{2}-\gamma_{3}\right) \\
& R_{3}=\left(\gamma_{3}^{2}+\gamma_{3} K+L\right) /\left(\gamma_{1}-\gamma_{3}\right)\left(\gamma_{2}-\gamma_{3}\right)
\end{aligned}
$$

$\mathrm{K}, \mathrm{L}$ and $\gamma_{1}, \gamma_{2}, \gamma_{3}$ are given in (6) \& (7) respectively.

The reliability expression given in (8) agrees with the result already developed by sagar et al [6]. For series system, we do not consider repairs, i.e $\mu_{0}=\mu_{1}=0$ in the model. Therefore, the successful operation is shown by state ' 0 ' of the model. By substituting $\gamma_{1}, \gamma_{2}$ and $\gamma_{3}$ as seen in (8), it can be simplified to

$R_{c h s}(t)=\exp \left[-\left(3 \lambda_{i} p_{I}+\lambda_{c} p_{2}+\lambda_{h} p_{3}\right) t\right]$

Therefore, the maximum likelihood estimation of the reliability function for series system is given by

$$
\hat{R}_{c h s}(t)=\exp \left(-\left(3 \hat{\bar{x}} p_{1}+\hat{\bar{y}} p_{2}+\hat{\bar{w}} p_{3}\right)\right)
$$

Where $\hat{\bar{x}}, \hat{\bar{y}} \& \hat{\bar{w}}$ are the samples estimates given in "section 5 ".

\subsection{Estimation of the Reliability function - Parallel system}

The reliability function for parallel system is obtained as

$$
\begin{aligned}
R_{c h p}(t) & =p_{0}(t)+p_{I}(t)+p_{2}(t) \\
& =J_{1} \exp \left(\gamma_{1} t\right)-J_{2} \exp \left(\gamma_{2} t\right)+J_{3} \exp \left(\gamma_{3} t\right)
\end{aligned}
$$

Where,

$$
\begin{aligned}
J_{I}= & {\left[\left(\gamma_{1}^{2}+\gamma_{1} K+L\right)+\left(3 \lambda_{i} p_{1}\right)\left(\gamma_{1}+\lambda_{i} p_{1}+2 \mu\right)+6\left(\lambda_{i} p_{1}\right)^{2}\right] / } \\
& \left(\gamma_{1}-\gamma_{2}\right)\left(\gamma_{1}-\gamma_{3}\right) \\
J_{2}= & {\left[\left(\gamma_{2}^{2}+\gamma_{2} K+L\right)+\left(3 \lambda_{i} p_{1}\right)\left(\gamma_{2}+\lambda_{i} p_{1}+2 \mu\right)+6\left(\lambda_{i} p_{1}\right)^{2}\right] / } \\
& \left(\gamma_{1}-\gamma_{2}\right)\left(\gamma_{2}-\gamma_{3}\right) \\
J_{3}= & {\left[\left(\gamma_{3}^{2}+\gamma_{3} K+L\right)+\left(3 \lambda_{i} p_{1}\right)\left(\gamma_{3}+\lambda_{i} p_{1}+2 \mu\right)+6\left(\lambda_{i} p_{1}\right)^{2}\right] / } \\
& \left(\gamma_{1}-\gamma_{3}\right)\left(\gamma_{2}-\gamma_{3}\right)
\end{aligned}
$$

$K, L$, and $\gamma_{1}, \gamma_{2}, \gamma_{3}$ are given in (6) and (7) respectively.

The reliability expression given in (11) agrees with the result already developed by Sagar et al [6]. Therefore, the maximum likelihood estimate of the reliability function for parallel system is given by

$$
\hat{R}_{c h p}(t)=J_{1}^{\prime} \exp \left(D_{1} t\right)-J_{2}^{\prime} \exp \left(D_{2} t\right)+J_{3}^{\prime} \exp \left(D_{3} t\right)
$$

Where,

$$
\begin{aligned}
& J_{1}^{\prime}=\frac{\left[\left(D_{1}^{2}+D_{1} K^{\prime}+L^{1}\right)+\left(3 \hat{\bar{x}} p_{1}\right)\left(D_{1}+\hat{\bar{x}} p_{1}+2 \hat{\bar{z}}\right)+6\left(\hat{\bar{x}} p_{1}\right)^{2}\right]}{\left(D_{1}-D_{2}\right)\left(D_{1}-D_{3}\right)} \\
& J_{2}^{\prime}=\frac{\left[\left(D_{2}^{2}+D_{2} K^{\prime}+L^{1}\right)+\left(3 \hat{\bar{x}} p_{1}\right)\left(D_{2}+\hat{\bar{x}} p_{1}+2 \hat{\bar{z}}\right)+6\left(\hat{\bar{x}} p_{1}\right)^{2}\right]}{\left(D_{1}-D_{2}\right)\left(D_{2}-D_{3}\right)} \\
& J_{3}^{\prime}=\frac{\left[\left(D_{3}^{2}+D_{3} K^{\prime}+L^{1}\right)+\left(3 \hat{\bar{x}} p_{1}\right)\left(D_{3}+\hat{\bar{x}} p_{1}+2 \hat{\bar{z}}\right)+6\left(\hat{\bar{x}} p_{1}\right)^{2}\right]}{\left(D_{1}-D_{3}\right)\left(D_{2}-D_{3}\right)}
\end{aligned}
$$

$\left.\begin{array}{l}K^{\prime}=\left(3 \hat{\bar{x}} p_{1}+3 \hat{\bar{z}}\right) \\ L^{\prime}=2\left(\hat{\bar{x}} p_{1}\right)^{2}+\hat{\bar{x}} p_{1} \hat{\bar{z}}+2(\hat{\bar{z}})^{2}\end{array}\right)$

$D_{1}=-D \sin \left(\alpha^{\prime}\right)-S_{1}^{\prime} / 3$

$D_{2}=D \sin \left(\pi / 3+\alpha^{\prime}\right)-S_{1}^{\prime} / 3$

$\left.D_{3}=D \sin \left(-\pi / 3+\alpha^{\prime}\right)-S_{1}^{\prime} / 3\right)$

Where,

$$
\begin{aligned}
& D=(2 / 3)\left(\left(S_{1}^{\prime}\right)^{2}-3 S_{2}^{\prime}\right)^{1 / 2} \\
& \alpha^{\prime}=\left(\sin ^{-1}\left(-4 q^{\prime} / D^{3}\right)\right) / 3 \\
& q^{\prime}=S_{3}^{\prime}-\left(S_{1}^{\prime} S_{2}^{\prime}\right) / 3+2\left(S_{1}^{\prime}\right)^{3} / 27 \\
& S_{1}^{\prime}=\left(6 \hat{\bar{x}} p_{1}+\hat{\bar{w}} p_{3}+\hat{\bar{y}} p_{2}+3 \hat{\bar{z}}\right) \\
& S_{2}^{\prime}=\left(11\left(\hat{\bar{x}} p_{1}\right)^{2}+3 \hat{\bar{x}} p_{1} \hat{\bar{w}} p_{3}+3 \hat{\bar{x}} p_{1} \hat{\bar{y}} p_{2}+7 \hat{\bar{x}} p_{1} \hat{\bar{z}}+3 \hat{\bar{w}} p_{3} \hat{\bar{z}}+3 \hat{\bar{y}} p_{2} \hat{\bar{z}}+2(\hat{\bar{z}})^{2}\right) \\
& S_{3}^{\prime}=\left(6\left(\hat{\overline{\bar{x}}} p_{1}\right)^{3}+2\left(\hat{\bar{x}} p_{1}\right)^{2}\left(\hat{\overline{\bar{w}}} p_{3}\right)+2\left(\hat{\overline{\bar{x}}} p_{1}\right)^{2}\left(\hat{\bar{y}} p_{2}\right)+2\left(\hat{\bar{y}} p_{2}\right)(\hat{\bar{z}})^{2}+\right) \\
& \left(\left(\hat{\bar{x}} p_{1}\right)\left(\hat{\bar{w}} p_{3}\right)(\hat{\bar{z}})+\left(\hat{\bar{x}} p_{1}\right)\left(\hat{\bar{y}} p_{2}\right)(\hat{\bar{z}})+2\left(\hat{\bar{w}} p_{3}\right)(\hat{\bar{z}})^{2}\right.
\end{aligned}
$$

And $\hat{\bar{x}}, \hat{\bar{y}}, \hat{\bar{w}} \& \hat{\bar{z}}$ are sample estimates given in "section 5". 


\subsection{Estimation of the MTBF function - Series system}

The mean time between failure function for series system is obtain as

$$
E_{c h s}(T)=\int_{0}^{\infty} R_{c h s}(t) \cdot d t
$$

Using the result in (9), the expression reduces to

$$
E_{c h s}(T)=1 /\left(3 \lambda_{i} p_{1}+\lambda_{c} p_{2}+\lambda_{h} p_{3}\right)
$$

The above expression given in (15) agrees with the result already developed by Sagar et al [6]. Therefore, the maximum likelihood estimate of mean time between failures function for series system is given by

$$
\hat{E}_{c h s}(T)=\frac{1}{\left(3 \hat{\bar{x}} p_{1}+\hat{\bar{y}} p_{2}+\hat{\bar{w}} p_{3}\right)}
$$

Where $\hat{\bar{x}}, \hat{\bar{y}} \& \hat{\bar{w}}$ are sample estimates given in "section 5".

\subsection{Estimation of the MTBF function - Parallel system}

The mean time between failure function for parallel system is obtained as

$$
E_{c h p}(T)=\int_{0}^{\infty} R_{c h p}(t) \cdot d t
$$

Using the result in (11), the expression reduces to

$$
E_{c h p}(T)=-\left[\left(6 \lambda_{i} p_{1} \mu+9\left(\lambda_{i} p_{1}\right)^{2}+L\right) /\left(\gamma_{1} \gamma_{2} \gamma_{3}\right)\right]
$$

Where $L$ and $\gamma_{1} \gamma_{2} \gamma_{3}$ are given in (6) \& (7) respectively.

The above expression given in (17) agrees with the result already developed by Sagar et al [6]. Therefore, the maximum likelihood estimate of mean time between failure function for parallel system is given by

$$
\hat{E}_{c h p}(T)=-\frac{\left(6 \hat{\bar{x}} p_{1} \hat{\bar{z}}+9\left(\hat{\bar{x}} p_{1}\right)^{2}+L^{\prime}\right)}{\left(D_{1} D_{2} D_{3}\right)}
$$

Where $L^{\prime}$ and $D_{1}, D_{2}, D_{3}$ are given in (13) and (14) respectively.

\section{SIMULATION STUDY}

In the present work, the M L estimates of reliability and MTBF (both series and parallel systems) were not identified with exact or analytical form of probability density function since they are complex functions of sample information. Hence an attempt is made to develop empirical evidence of M L estimation approach by Monte-Carlo Simulation using an appropriate computer package for validity of results.

For a range of specified values of the rates of individual $\left(\lambda_{i}\right)$, CCS failures $\left(\lambda_{c}\right)$, human errors $\left(\lambda_{h}\right)$ and service rate $(\mu)$ and for the samples of sizes $n=5(5) 30$ were simulated in each case with $\mathrm{N}=10,000(20,000) 90,000$ in order to evolve Mean Square Error (MSE) in each case. For large samples M L estimators are undisputedly better since they are CAN estimators. Interestingly, our simulation study shows that the M L estimate is still reasonably good giving near accurate estimate even for a sample size as low as five (i.e $n=5)$. This shows that M L approach and estimators are quite useful in estimating reliability indices.

\subsection{Numerical Illustration}

\section{Table 6.1}

Reliability function for three component identical Series system with $\lambda_{\mathrm{i}}=0.1 ; \lambda_{\mathrm{c}}=0.2 ; \lambda_{\mathrm{h}}=0.3 ; \mathrm{p}_{1}=0.5 ; \mathrm{p}_{2}=0.25$; $\mathrm{p}_{3}=0.25 ; \mathbf{t}=\mathbf{1}$

\begin{tabular}{|c|c|c|c|}
\hline \multicolumn{4}{|c|}{ Sample size $\mathrm{n}=5$} \\
\hline $\mathrm{N}$ & $R_{\text {chs }}(t)$ & $\hat{R}_{\text {chs }}(t)$ & M S E \\
\hline 10000 & 0.759572 & 0.689115 & 0.013600 \\
\hline 30000 & 0.759572 & 0.689573 & 0.013368 \\
\hline 50000 & 0.759572 & 0.688885 & 0.013543 \\
\hline 70000 & 0.759572 & 0.689220 & 0.013480 \\
\hline 90000 & 0.759572 & 0.689261 & 0.013440 \\
\hline
\end{tabular}

\begin{tabular}{|c|c|c|c|}
\hline \multicolumn{4}{|c|}{ Sample size $\mathrm{n}=10$} \\
\hline $\mathrm{N}$ & $R_{\text {chs }}(t)$ & $\hat{R}_{\text {chs }}(t)$ & M S E \\
\hline 10000 & 0.759572 & 0.717531 & 0.004967 \\
\hline 30000 & 0.759572 & 0.718588 & 0.004788 \\
\hline 50000 & 0.759572 & 0.718101 & 0.004777 \\
\hline 70000 & 0.759572 & 0.718454 & 0.004813 \\
\hline 90000 & 0.759572 & 0.718201 & 0.004831 \\
\hline
\end{tabular}

\begin{tabular}{|c|c|c|c|}
\hline \multicolumn{4}{|c|}{ Sample size $\mathrm{n}=15$} \\
\hline $\mathrm{N}$ & $R_{\text {chs }}(t)$ & $\hat{R}_{\text {chs }}(t)$ & M S E \\
\hline 10000 & 0.759572 & 0.727730 & 0.002836 \\
\hline 30000 & 0.759572 & 0.727201 & 0.002877 \\
\hline 50000 & 0.759572 & 0.726804 & 0.002933 \\
\hline 70000 & 0.759572 & 0.727051 & 0.002898 \\
\hline 90000 & 0.759572 & 0.727030 & 0.002929 \\
\hline
\end{tabular}

\begin{tabular}{|c|c|c|c|}
\hline \multicolumn{4}{|c|}{ Sample size $\mathrm{n}=20$} \\
\hline $\mathrm{N}$ & $R_{\text {chs }}(t)$ & $\hat{R}_{\text {chs }}(t)$ & M S E \\
\hline 10000 & 0.759572 & 0.731413 & 0.002082 \\
\hline 30000 & 0.759572 & 0.731192 & 0.002126 \\
\hline 50000 & 0.759572 & 0.731460 & 0.002097 \\
\hline 70000 & 0.759572 & 0.731542 & 0.002108 \\
\hline 90000 & 0.759572 & 0.731472 & 0.002100 \\
\hline
\end{tabular}




\begin{tabular}{|c|c|c|c|}
\hline \multicolumn{4}{|c|}{ Sample size $\mathrm{n}=25$} \\
\hline $\mathrm{N}$ & $R_{\text {chs }}(t)$ & $\hat{R}_{\text {chs }}(t)$ & M S E \\
\hline 10000 & 0.759572 & 0.733631 & 0.001708 \\
\hline 30000 & 0.759572 & 0.733851 & 0.001673 \\
\hline 50000 & 0.759572 & 0.733953 & 0.001668 \\
\hline 70000 & 0.759572 & 0.733852 & 0.001677 \\
\hline 90000 & 0.759572 & 0.733986 & 0.001664 \\
\hline
\end{tabular}

\begin{tabular}{|c|c|c|c|}
\hline \multicolumn{4}{|c|}{ Sample size $\mathrm{n}=20$} \\
\hline $\mathrm{N}$ & $R_{\text {chp }}(t)$ & $\hat{R}_{\text {chp }}(t)$ & M S E \\
\hline 10000 & 0.887175 & 0.873056 & 0.000657 \\
\hline 30000 & 0.887175 & 0.873026 & 0.000662 \\
\hline 50000 & 0.887175 & 0.872856 & 0.000667 \\
\hline 70000 & 0.887175 & 0.872931 & 0.000667 \\
\hline 90000 & 0.887175 & 0.872983 & 0.000655 \\
\hline
\end{tabular}

\begin{tabular}{|c|c|c|c|}
\hline \multicolumn{4}{|c|}{ Sample size $\mathrm{n}=30$} \\
\hline $\mathrm{N}$ & $R_{\text {chs }}(t)$ & $\hat{R}_{\text {chs }}(t)$ & M S E \\
\hline 10000 & 0.759572 & 0.735627 & 0.001394 \\
\hline 30000 & 0.759572 & 0.735225 & 0.001419 \\
\hline 50000 & 0.759572 & 0.735542 & 0.001399 \\
\hline 70000 & 0.759572 & 0.735537 & 0.001404 \\
\hline 90000 & 0.759572 & 0.735497 & 0.001410 \\
\hline
\end{tabular}

\begin{tabular}{|c|c|c|c|}
\hline \multicolumn{4}{|c|}{ Sample size $\mathrm{n}=25$} \\
\hline $\mathrm{N}$ & $R_{\text {chp }}(t)$ & $\hat{R}_{\text {chp }}(t)$ & M S E \\
\hline 10000 & 0.887175 & 0.874175 & 0.000522 \\
\hline 30000 & 0.887175 & 0.874230 & 0.000523 \\
\hline 50000 & 0.887175 & 0.874086 & 0.000527 \\
\hline 70000 & 0.887175 & 0.874210 & 0.000521 \\
\hline 90000 & 0.887175 & 0.874168 & 0.000519 \\
\hline
\end{tabular}

\section{Table 6.2}

Reliability function for three component identical Parallel system with $\lambda_{\mathrm{i}}=0.1 ; \lambda_{\mathrm{c}}=0.2 ; \lambda_{\mathrm{h}}=0.3 ; \mathrm{p}_{1}=0.5 ; \mathrm{p}_{2}=0.25$; $\mathrm{p}_{3}=0.25 ; \mu=0.5 ; \quad \mathbf{t}=\mathbf{1}$

\begin{tabular}{|c|c|c|c|}
\hline \multicolumn{4}{|c|}{ Sample size $\mathrm{n}=5$} \\
\hline $\mathrm{N}$ & $R_{\text {chp }}(t)$ & $\hat{R}_{\text {chp }}(t)$ & M S E \\
\hline 10000 & 0.887175 & 0.849740 & 0.004932 \\
\hline 30000 & 0.887175 & 0.850022 & 0.004915 \\
\hline 50000 & 0.887175 & 0.850103 & 0.004870 \\
\hline 70000 & 0.887175 & 0.850430 & 0.004762 \\
\hline 90000 & 0.887175 & 0.849990 & 0.004864 \\
\hline
\end{tabular}

\begin{tabular}{|c|c|c|c|}
\hline \multicolumn{4}{|c|}{ Sample size $\mathrm{n}=10$} \\
\hline $\mathrm{N}$ & $R_{\text {chp }}(t)$ & $\hat{R}_{c h p}(t)$ & M S E \\
\hline 10000 & 0.887175 & 0.865974 & 0.001597 \\
\hline 30000 & 0.887175 & 0.865919 & 0.001601 \\
\hline 50000 & 0.887175 & 0.865943 & 0.001606 \\
\hline 70000 & 0.887175 & 0.865985 & 0.001584 \\
\hline 90000 & 0.887175 & 0.865867 & 0.001597 \\
\hline
\end{tabular}

\begin{tabular}{|c|c|c|c|}
\hline \multicolumn{4}{|c|}{ Sample size $\mathrm{n}=30$} \\
\hline $\mathrm{N}$ & $R_{\text {chp }}(t)$ & $\hat{R}_{\text {chp }}(t)$ & M S E \\
\hline 10000 & 0.887175 & 0.875058 & 0.000436 \\
\hline 30000 & 0.887175 & 0.875115 & 0.000430 \\
\hline 50000 & 0.887175 & 0.875075 & 0.000435 \\
\hline 70000 & 0.887175 & 0.874990 & 0.000438 \\
\hline 90000 & 0.887175 & 0.874957 & 0.000438 \\
\hline
\end{tabular}

\section{Table 6.3}

Simulation results for Mean Time Between Failures function Series System with $\lambda_{\mathrm{i}}=0.5 ; \quad \lambda_{\mathrm{c}}=0.6 ; \quad \lambda_{\mathrm{h}}=0.7 ; \quad \mathrm{p}_{1}=0.5$; $\mathrm{p}_{2}=0.25 ; \mathrm{p}_{3}=0.25$

\begin{tabular}{|c|c|c|c|}
\hline \multicolumn{4}{|c|}{ Sample size $\mathrm{n}=5$} \\
\hline $\mathrm{N}$ & $E_{\text {chs }}(T)$ & $\hat{E}_{\text {chs }}(T)$ & M S E \\
\hline 10000 & 0.930233 & 0.775375 & 0.100702 \\
\hline 30000 & 0.930233 & 0.774135 & 0.098398 \\
\hline 50000 & 0.930233 & 0.772012 & 0.099046 \\
\hline 70000 & 0.930233 & 0.773719 & 0.099438 \\
\hline 90000 & 0.930233 & 0.773195 & 0.099385 \\
\hline
\end{tabular}

\begin{tabular}{|c|c|c|c|}
\hline \multicolumn{4}{|c|}{ Sample size $\mathrm{n}=15$} \\
\hline $\mathrm{N}$ & $R_{\text {chp }}(t)$ & $\hat{R}_{\text {chp }}(t)$ & M S E \\
\hline 10000 & 0.887175 & 0.870512 & 0.000914 \\
\hline 30000 & 0.887175 & 0.870614 & 0.000928 \\
\hline 50000 & 0.887175 & 0.870613 & 0.000941 \\
\hline 70000 & 0.887175 & 0.870641 & 0.000932 \\
\hline 90000 & 0.887175 & 0.870702 & 0.000934 \\
\hline
\end{tabular}

\begin{tabular}{|c|c|c|c|}
\hline \multicolumn{4}{|c|}{ Sample size $\mathrm{n}=10$} \\
\hline $\mathrm{N}$ & $E_{c h s}(T)$ & $\hat{E}_{c h s}(T)$ & M S E \\
\hline 10000 & 0.930233 & 0.815011 & 0.053551 \\
\hline 30000 & 0.930233 & 0.818934 & 0.053011 \\
\hline 50000 & 0.930233 & 0.817060 & 0.052856 \\
\hline 70000 & 0.930233 & 0.818580 & 0.053243 \\
\hline 90000 & 0.930233 & 0.817579 & 0.053208 \\
\hline
\end{tabular}




\begin{tabular}{|c|c|c|c|}
\hline \multicolumn{4}{|c|}{ Sample size $\mathrm{n}=15$} \\
\hline $\mathrm{N}$ & $E_{\text {chs }}(T)$ & $\hat{E}_{\text {chs }}(T)$ & M S E \\
\hline 10000 & 0.930233 & 0.835785 & 0.036667 \\
\hline 30000 & 0.930233 & 0.833126 & 0.037403 \\
\hline 50000 & 0.930233 & 0.831677 & 0.037694 \\
\hline 70000 & 0.930233 & 0.832914 & 0.037361 \\
\hline 90000 & 0.930233 & 0.832948 & 0.037576 \\
\hline
\end{tabular}

\begin{tabular}{|c|c|c|c|}
\hline \multicolumn{4}{|c|}{ Sample size $\mathrm{n}=10$} \\
\hline $\mathrm{N}$ & $E_{c h p}(T)$ & $\hat{E}_{c h p}(T)$ & M S E \\
\hline 10000 & 1.169361 & 1.052237 & 0.093493 \\
\hline 30000 & 1.169361 & 1.050642 & 0.093152 \\
\hline 50000 & 1.169361 & 1.051699 & 0.093652 \\
\hline 70000 & 1.169361 & 1.051567 & 0.093196 \\
\hline 90000 & 1.169361 & 1.050292 & 0.093135 \\
\hline
\end{tabular}

\begin{tabular}{|c|c|c|c|}
\hline \multicolumn{4}{|c|}{ Sample size $\mathrm{n}=20$} \\
\hline $\mathrm{N}$ & $E_{\text {chs }}(T)$ & $\hat{E}_{\text {chs }}(T)$ & M S E \\
\hline 10000 & 0.930233 & 0.839737 & 0.029085 \\
\hline 30000 & 0.930233 & 0.840229 & 0.029468 \\
\hline 50000 & 0.930233 & 0.840799 & 0.029124 \\
\hline 70000 & 0.930233 & 0.841224 & 0.029375 \\
\hline 90000 & 0.930233 & 0.840687 & 0.029251 \\
\hline
\end{tabular}

\begin{tabular}{|c|c|c|c|}
\hline \multicolumn{4}{|c|}{ Sample size $\mathrm{n}=15$} \\
\hline $\mathrm{N}$ & $E_{c h p}(T)$ & $\hat{E}_{c h p}(T)$ & M S E \\
\hline 10000 & 1.169361 & 1.059674 & 0.063832 \\
\hline 30000 & 1.169361 & 1.060975 & 0.063887 \\
\hline 50000 & 1.169361 & 1.061927 & 0.064016 \\
\hline 70000 & 1.169361 & 1.061602 & 0.064046 \\
\hline 90000 & 1.169361 & 1.062657 & 0.064234 \\
\hline
\end{tabular}

\begin{tabular}{|c|c|c|c|}
\hline \multicolumn{4}{|c|}{ Sample size $\mathrm{n}=25$} \\
\hline $\mathrm{N}$ & $E_{\text {chs }}(T)$ & $\hat{E}_{\text {chs }}(T)$ & M S E \\
\hline 10000 & 0.930233 & 0.843980 & 0.024777 \\
\hline 30000 & 0.930233 & 0.844510 & 0.024431 \\
\hline 50000 & 0.930233 & 0.845286 & 0.024315 \\
\hline 70000 & 0.930233 & 0.844804 & 0.024478 \\
\hline 90000 & 0.930233 & 0.845343 & 0.024297 \\
\hline
\end{tabular}

\begin{tabular}{|c|c|c|c|}
\hline \multicolumn{4}{|c|}{ Sample size $\mathrm{n}=30$} \\
\hline $\mathrm{N}$ & $E_{\text {chs }}(T)$ & $\hat{E}_{\text {chs }}(T)$ & M S E \\
\hline 10000 & 0.930233 & 0.848411 & 0.021064 \\
\hline 30000 & 0.930233 & 0.846535 & 0.021355 \\
\hline 50000 & 0.930233 & 0.848110 & 0.021092 \\
\hline 70000 & 0.930233 & 0.847995 & 0.021174 \\
\hline 90000 & 0.930233 & 0.847722 & 0.021248 \\
\hline
\end{tabular}

\begin{tabular}{|c|c|c|c|}
\hline \multicolumn{4}{|c|}{ Sample size $\mathrm{n}=20$} \\
\hline $\mathrm{N}$ & $E_{c h p}(T)$ & $\hat{E}_{c h p}(T)$ & M S E \\
\hline 10000 & 1.169361 & 1.069577 & 0.049401 \\
\hline 30000 & 1.169361 & 1.068467 & 0.049425 \\
\hline 50000 & 1.169361 & 1.067072 & 0.049651 \\
\hline 70000 & 1.169361 & 1.067911 & 0.049747 \\
\hline 90000 & 1.169361 & 1.068214 & 0.049237 \\
\hline
\end{tabular}

\begin{tabular}{|c|c|c|c|}
\hline \multicolumn{5}{|c|}{ Sample size $\mathrm{n}=25$} \\
\hline $\mathrm{N}$ & $E_{c h p}(T)$ & $\hat{E}_{c h p}(T)$ & M S E \\
\hline 10000 & 1.169361 & 1.070323 & 0.040910 \\
\hline 30000 & 1.169361 & 1.070973 & 0.041083 \\
\hline 50000 & 1.169361 & 1.069865 & 0.041186 \\
\hline 70000 & 1.169361 & 1.070826 & 0.040923 \\
\hline 90000 & 1.169361 & 1.070431 & 0.040869 \\
\hline
\end{tabular}

\section{Table 6.4}

Simulation results for Mean Time Between Failures function Parallel System with $\lambda_{\mathrm{i}}=0.5 ; \lambda_{\mathrm{c}}=1.5 ; \lambda_{\mathrm{h}}=2 ; \mathrm{p}_{1}=0.5$; $\mathrm{p}_{2}=0.25 ; \mathrm{p}_{3}=0.25 ; \mu=5$

\begin{tabular}{|c|c|c|c|}
\hline \multicolumn{4}{|c|}{ Sample size $\mathrm{n}=5$} \\
\hline $\mathrm{N}$ & $E_{c h p}(T)$ & $\hat{E}_{c h p}(T)$ & M S E \\
\hline 10000 & 1.169361 & 1.020356 & 0.178590 \\
\hline 30000 & 1.169361 & 1.024095 & 0.180666 \\
\hline 50000 & 1.169361 & 1.021380 & 0.179231 \\
\hline 70000 & 1.169361 & 1.023405 & 0.178946 \\
\hline 90000 & 1.169361 & 1.021091 & 0.180232 \\
\hline
\end{tabular}

\begin{tabular}{|c|c|c|c|}
\hline \multicolumn{4}{|c|}{ Sample size $\mathrm{n}=30$} \\
\hline $\mathrm{N}$ & $E_{\text {chp }}(T)$ & $\hat{E}_{\text {chp }}(T)$ & M S E \\
\hline 10000 & 1.169361 & 1.072988 & 0.035464 \\
\hline 30000 & 1.169361 & 1.073722 & 0.035274 \\
\hline 50000 & 1.169361 & 1.073574 & 0.035206 \\
\hline 70000 & 1.169361 & 1.072761 & 0.035357 \\
\hline 90000 & 1.169361 & 1.072214 & 0.035439 \\
\hline
\end{tabular}

\section{CONCLUSIONS}

The M L estimates of reliability measures like reliability function $[R(t)]$ and MTBF $[E(T)]$ for both series and parallel systems of the present model were obtained. The empirical evidence was developed by using Monte-Carlo Simulation for selected values of the failure and repair rates to establish the validity and precision of the M L estimates of the above said reliability measures. The simulation results suggest that the $\mathrm{M}$ L estimate is reasonably very good and give accurate estimates even for sample size $n=5$. For all the cases of estimates mean 
square error is almost zero with sample sizes ' $n$ ' tending to large i.e 20 and above.

Therefore, the M L estimators are generally hard to beat consistently, even in small samples and our simulation results showed a strong preference for the M L estimation method for situations arising in practical reliability analysis.

\section{REFERENCES}

[1] Atwood, C. L. (1986) "The binomial failure rate common cause model" in Technometrics, vol: 28 , pp. 139-147.

[2] Billinton, R \& Allan, R. N (1983) "Reliability Evaluation of Engineering Systems; Concepts and Techniques", Plenum Press, New York.

[3] Chari, A. A., Sastry, M. P and Madhusudhana Verma, S. (1991) "Reliability analysis in the presence of common cause shock failures", Micro-Electronics and reliability,31, pp.15-19.

[4] Reddy, Y. R. (2003) "Reliability analysis for two unit non-identical system with CCS failures", Ph.D thesis, S.K.University, Anantapur.

[5] Ritika Wason, Ahmed. P and Qasim Rafiq, M. (2012), "New paradigm for software reliability estimation", International journal of computer application, vol.44, No.14, pp. 39-44.

[6] Sagar, G. Y., Reddy, Y. R., Umashankar, C and Verma, S. M. (2010), " Some reliability measures for three component system in the presence of CCS failures and human errors", Journal of interdisciplinary mathematics, vol.13, No. 2, pp. 143-151. 